\title{
Batching: A Design Pattern for Efficient and Flexible Client/Server Interaction
}

\author{
Francisco J. Ballesteros ${ }^{1}$, Fabio Kon ${ }^{2}$, Marta Patiño ${ }^{3}$, Ricardo Jiménez ${ }^{3}$, \\ Sergio Arévalo ${ }^{1}$, and Roy H. Campbell ${ }^{4}$ \\ 1 University Rey Juan Carlos \\ nemo@lsub.org \\ 2 University of São Paulo \\ kon@ime.usp.br \\ 3 Technical University of Madrid \\ 4 University of Illinois at Urbana-Champaign
}

\begin{abstract}
The BATCHING design pattern consists of a common piece of design and implementation that is shared by a wide variety of well-known techniques in Computing such as gather/scatter for input/output, code downloading for system extension, message batching, mobile agents, and deferred calls for disconnected operation.

All techniques mentioned above are designed for applications running across multiple domains (e.g., multiple processes or multiple nodes in a network). In these techniques, multiple operations are bundled together and then sent to a different domain, where they are executed. In some cases, the objective is to reduce the number of domain-crossings. In other cases, it is to enable dynamic server extension.

In this article, we present the BATCHING pattern, discuss the circumstances in which the pattern should and should not be used, and identify eight classes of existing techniques that instantiate it.
\end{abstract}

\section{Introduction}

Applications such as code downloading, message batching, gather/scatter, and mobile agents follow the client/server model of interaction. A closer look reveals that all of them group a set of operations, and submit them to a server for execution. The submission of operations aims at reducing domain-crossings and/or enable dynamic server extension. For instance, code downloading into operating system kernels intends to save domain-crossings and, at the same time, enable system extension. Message batching and mobile agents intend to save domaincrossings.

Consider a program using a file server such as the one in Figure 1 . In a typical client/server interaction, the client sends a command (read or write) to the server, waits for the reply, and then continues.

Suppose that read and write are handled by the same server and that crossdomain calls (i.e., calls from the client to the server) are much heavier than calls made within the server. In this case, it will be much more efficient to send the entire while loop to the file server for execution. 


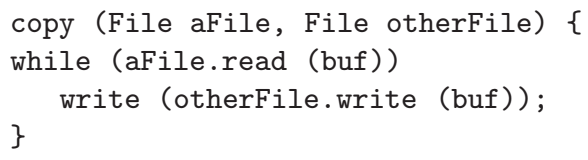

Fig. 1. File copy code
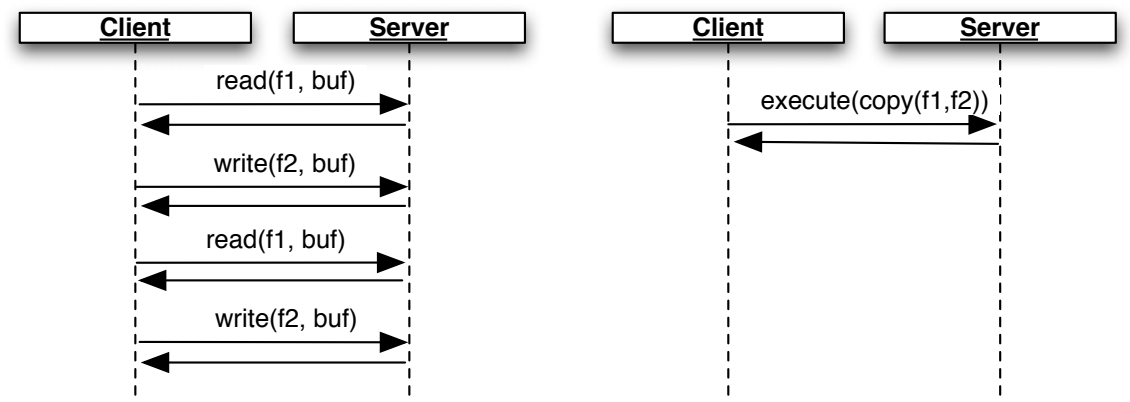

Fig. 2. Interactions corresponding to read/write services and a copy service

Instead of having multiple cross-domain calls, as depicted in the left-hand side of Figure 2, a single call suffices if the client sends the code to the server for execution, as shown in right-hand side of Figure 2. To do so, it is necessary to extend the file server to support the execution of programs submitted by different clients.

\section{The Problem}

Both cross-domain data traffic and cross-domain call latency have a significant impact on the efficiency of multi-domain applications. Cross-domain calls and cross-domain data transfers also happen on centralized environments. For instance, almost every operating system has a domain boundary between user space and kernel space (both entering and leaving the kernel requires a domain crossing). An application using multiple processes has a domain boundary between every two of its processes. Besides, in a distributed system, the network behaves as a domain boundary.

The line separating two different domains has to be considered while designing the application. There are two main issues causing problems to any application crossing the line: data movement and call latency.

Within a protection domain (e.g., a Unix process), an object can pass data efficiently to any other object. For passing a large amount of data, a reference can be used. However, whenever an object has to pass data to another object in a different domain, data has to be copied. Although some zero-copy networking frameworks avoid data copying within a single node in a network, data still has to be "copied" through the network in distributed applications. 
In many domains, such as file systems and databases, data movement can be the major performance bottleneck. Therefore, avoiding unnecessary data transfer operations may be crucial. Under many circumstances, unnecessary data transfers occur just because the object controlling the operation resides far from the data source or sink. That is precisely what happens in the file copy example in the previous section: the client object performing the copy and the file server objects were placed in different domains. Thus, data came to the client just to go back to the server.

Another issue is call latency. A call between two objects residing in different domains takes much more time to complete than a typical method call within a single domain. The reason is simply that a domain boundary has to be crossed; that usually involves either crossing the operating system kernel interface (in a single node), network messaging (in a distributed environment), or both. Therefore, avoiding domain crossing when performing calls is crucial for performance. Any solution reducing the number of domain crossings can make the application run faster.

When designing a solution, it should be taken into account that, under certain circumstances (e.g., when inexpensive domain crossing is available and efficiency is the primary objective), the overhead introduced to solve the problem might actually degrade performance. However, even when cheap domain crossing is available, the overhead caused by cross-domain data transfers (e.g., copying data or sending messages over a network) might still cause a performance problem.

Any solution must take into account carefully what is the real penalty caused by data copying and call latency. Also, this solution should be employed only when the overhead it causes is small compared to the penalties it avoids.

\section{The Solution}

\section{Batching, also known as CompositeCALL.}

By batching separate method calls, i.e., transforming them into a single crossdomain call, one can avoid unnecessary data copying and reduce the number of cross-domain calls. Clients can build a program (a "batch call") and transfer it to the server at once. The program performs multiple operations on that server even though the client had to send it only once.

In our example (see Figure 2, the interactions for copy), if BATCHING is not used, the file content has to travel twice across the network. When a copy program is submitted to the server, however, the file does not leave the server, it is copied locally. It behaves as if we had extended the server functionality dynamically by adding support for a copy operation.

\section{Pattern Structure}

In the following, we present the general structure of the pattern in its more complete and sophisticated form. Specific instances of the pattern often apply simplified implementations of the pattern. In Section 4.2, we describe the application of the pattern to the file service domain. 


\subsection{Participants}

The class hierarchy corresponding to the BATCHING pattern is shown in Figure3.

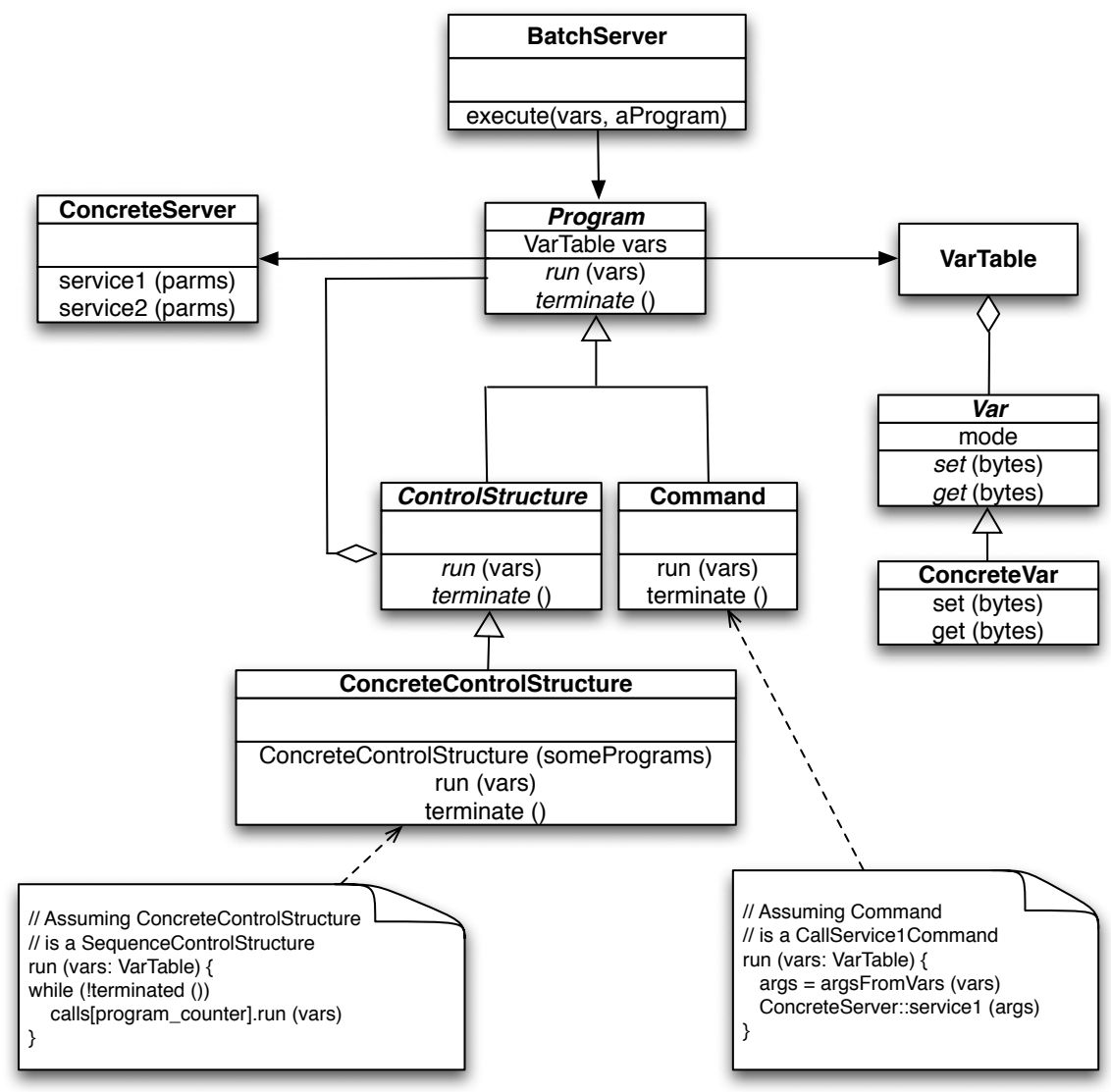

Fig. 3. BAtChing

BatchServer behaves as a FAÇADE GHJV95] to services provided by the server. An object of this class is located on the server side. It supplies interpretative facilities to service callers, so that clients can send a program to the server side instead of making direct calls to the server. The execute method is an entry point to the interpreter GHJV95, which interprets the "batch" program and returns its results to the client.

ConcreteServer is only present on the server side; it provides the set of entry points that can be called by the client.

Note that the ConcreteServer is actually the class (or the set of classes) one has on the server side before instantiating the pattern. It is mentioned here for completeness. 
Program is an abstract class that represents the batched calls or program to be interpreted. Clients build Program instances and send them to the BatchServer for execution. It is also responsible for maintaining an associated table of variables. The run method of a Program class implements the interpreter needed to run it on the server.

The Program is also responsible for performing proper program termination when an error occurs. The terminate method is provided as an abstract interface for program termination.

An alternate name for BAtching is CompositeCALL since Program and the following couple of classes form an instance of the COMPOSITE pattern GHJV95.

ControlStructure is a construct made of Programs. Its purpose is to bundle several Programs together according to some control structure (e.g., sequence, iteration, etc.).

ConcreteControlStructures represent structures such as conditionals, while constructs, sequences, etc. At the server side, this class is responsible for executing the concrete control structure represented by the class. ConcreteControlStructure constructors can be used at the client side to build complex Programs.

Command is a Program that represents a single operation; it resembles the CoMMAND pattern shown in GHJV95, hence the name. Examples of concrete Commands are arithmetic operations, logic operations, or calls to ConcreteServer entry points. The only purpose of BATCHInG is to bundle several concrete Commands together using ConcreteControlStructures.

VarTable stores the variables (i.e., the state) of the Program. It provides local storage and also holds any input parameter for the program. Output values from the program are also kept within the VarTable. The table is built at the client using the set of input parameters for the Program. Then, it is used within the server, while the Program is interpreted. The table is finally returned back to the user after completion of the Program.

There is a variable table per Program (pairs of VarTable and Program are sent together to the BatchServer). Thus, all components of a concrete Program share a single variable table so that they can share variables.

Var is an abstract class representing a variable of the program sent to the server. It has some associated storage (bytes, in Figure 3). Var instances are kept within a VarTable. Variables have a mode, which can be either in (parameter given to the Program), out (result to be given to the user), inout (both), or local (local variable). By including the mode qualifier, this class can be used for local variables as well as for input/output parameters.

ConcreteVar is a variable of a concrete type (integer, character, etc.). Its constructor is used at the client to declare variables or parameters to be used by the Program. At the server side, instances of this class are responsible for handling single, concrete pieces of data used by the program.

Note that in a concrete instance of the pattern, the Program (and related classes) may differ from the ones shown. That is, the core of the pattern includes BatchServer, Program, and ConcreteServer classes; but other classes 
shown here may differ depending on how the pattern is instantiated. The structure shown here is a general form for the pattern and can be directly applied to any particular implementation. However, to solve a concrete problem where the pattern applies, this structure can be modified and a Program class might be implemented in quite different ways depending on the particular form for the "program" sent from the client to the server. Doing so would also require changes to BatchServer because it must be able to process the program.

For example, all classes used by a client to build a program might be compiled to a bytecode representation of the program, to be sent to the server. In this case the BatchServer would be actually a bytecode interpreter. As another example, serialized forms of the program structures shown in this paper could be directly sent to the server, and BatchServer would simply use them after unpacking to interpret the program. More radical examples exist, such as making Program use a textual representation of the program and BatchServer, a compiler or

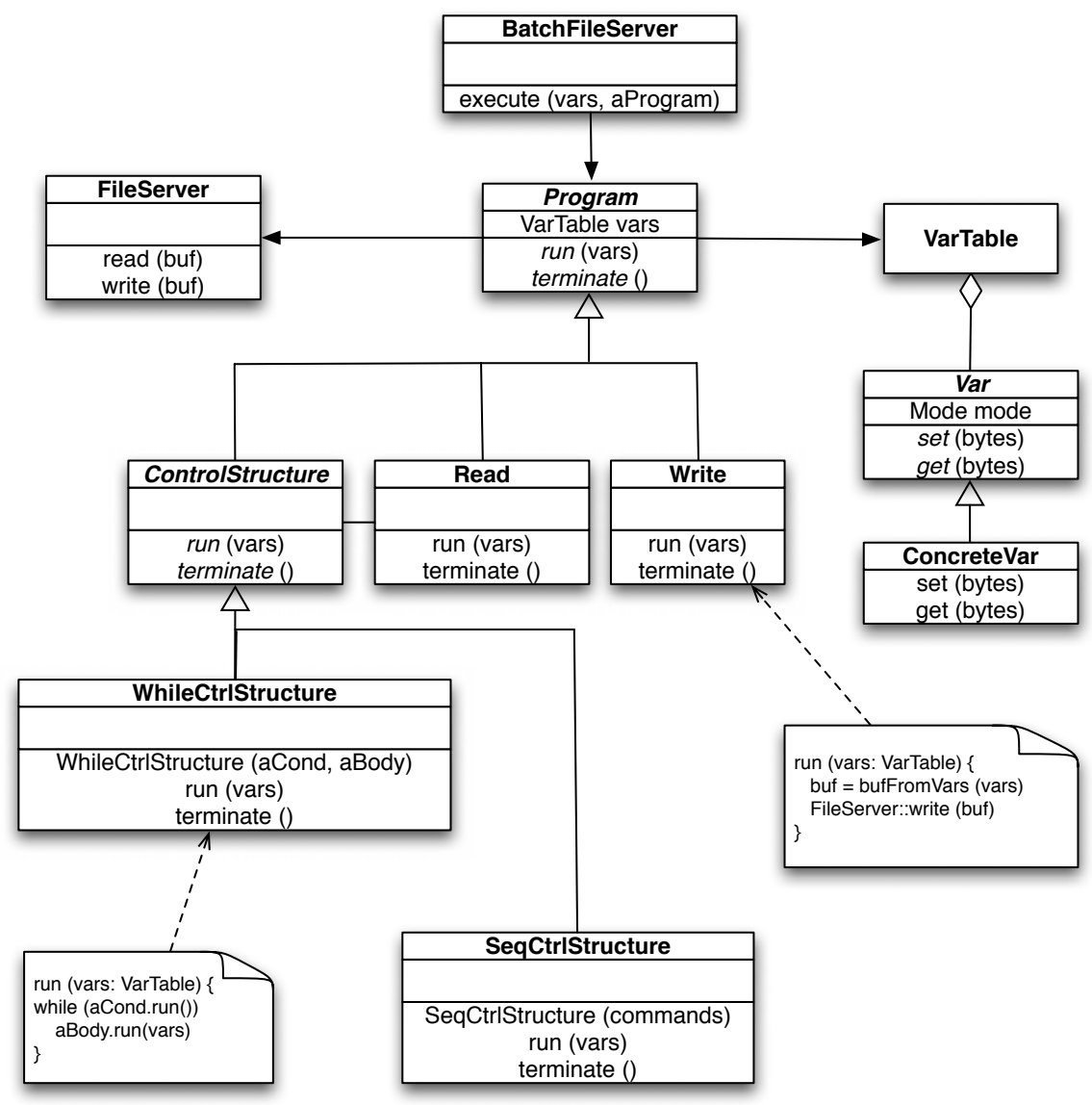

Fig. 4. BATChing File server 
interpreter for such "source"; or yet making Program use a binary representation of the program and implementing BatchServer simply as a call to the binary code received from the client.

All this said, in general, the structure shown for the pattern suffices and works well to solve the problem addressed by this pattern. In what follows, we use it in all our examples, but one should be aware that there are other useful variants of the pattern that use other forms of programs.

\subsection{The Pattern Applied to a File Server}

The concrete structure of classes for our file server example is shown in Figure 4 Intuitively, this BATCHING instance simply adds an interpreter (see the INTERPRETER pattern in GHJV95]) to the file server. That interpreter can execute programs that (1) call read and write and (2) can use while as a control structure.

We took as a starting point the FileServer class, which provides both read and write methods that operate on a file. We simplified the typical interface provided by a file server; a typical file server would contain several File objects that would supply read and write methods. To illustrate the pattern in a simple way, we omitted the file being used 1 .

The BatchFileServer is co-located with the FileServer, providing a new execute service that supplies an interpreted version of FileServer services. The BatchFileServer corresponds to the BatchServer in the pattern (see the pattern diagram in Figure 3).

The BatchFileServer accepts a Program, which is built in terms of ControlStructures and Read and Write commands.

To execute

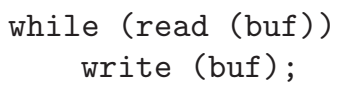

the Program sent to the BatchFileServer must be made of a WhileCtrlStructure, using a Read as the condition. The body for the WhileCtrlStructure must be a sequence made of a single Write command.

Here, WhileCtrlStructure and SeqCtrlStructure correspond to ConcreteControlStructures in the pattern. Read and Write match Commands in the pattern. The buffer used in the read and write operations is handled by a BufferVar class instance, corresponding to a ConcreteVar in the pattern.

A client can build a program (accessing the file server) by using constructors provided by WhileCtrlStructure, SeqCtrlStructure, Read, and Write. The client can later submit this batched call to the BatchFileServer execute method.

${ }^{1}$ Obtaining a complete implementation is a matter of adding a File class and adding file parameters to the read and write methods. 


\section{Dynamics}

The client builds a program (a "script" of commands) and sends it to the server, which interprets it. When the server receives a program, it first deserializes it. The interaction that follows is shown in Figure 5 .

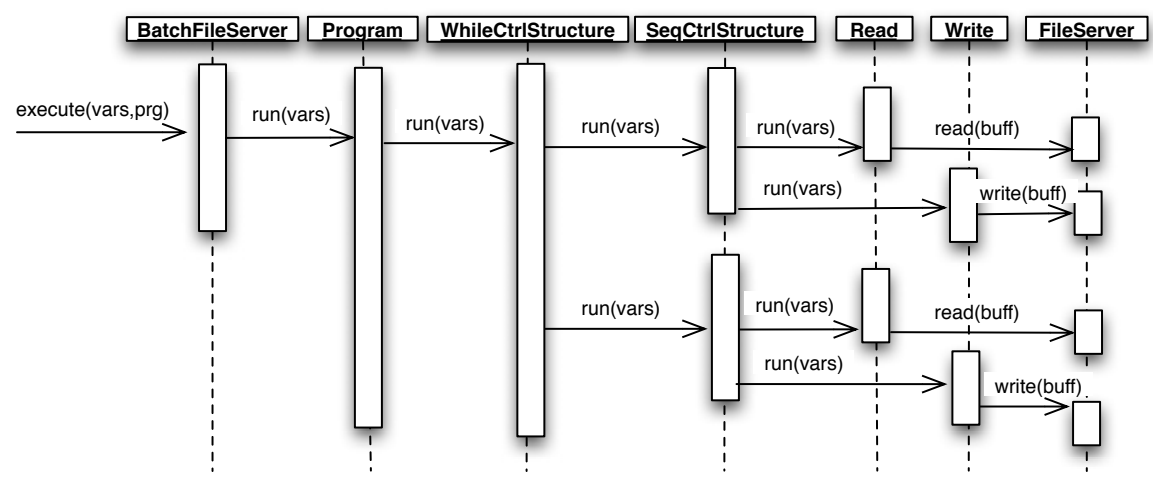

Fig. 5. Interaction diagram for a copy program

A BatchFileServer object at the server side is in charge of interpreting client programs. When its execute method is called, a program and a table of variables must be supplied. The execute method calls the run method of the program providing the table of variables; this method interprets the "batch" program. Once execute finishes, the results are returned.

The actual dynamics resulting from executing the "batch" program depends on the actual form used for the program. In our suggested form, the run method of the Program class implements recursive interpretation. When the program has been interpreted, that is, the run method has finished, the results of the program execution are still in the variable table. As part of the execute method, the table is serialized and sent back to the client.

Figure 5 shows the interaction diagram for our example copy batch program (with calls to Open and Close suppressed for the sake of simplicity). The run method of the Program calls the run method of the ConcreteControlStructure representing the program (the WhileCtrlStructure in the interaction diagram). ControlStructures provide a run method to interpret themselves. That is, a program has built-in its own interpreter, an instance of the INTERPRETER pattern GHJV95. So, the While command calls the run method of its inner component (SeqCtrlStructure in the interaction diagram for copy).

\section{Implementation Issues}

We now discuss two important aspects of using BATCHING: how to build programs for BATCHING and what to do when they fail. 


\subsection{Composing Programs}

How to build a program depends on the structure used for it. As an example, we use in this section the form suggested for programs in the previous description of the pattern. Readers should be aware, however, that other forms of implementing the pattern do exist.

Programs are made of statements and variables. In a BATCHING Program, each statement corresponds to a ConcreteControlStructure or concrete Command. Variables are instances of a ConcreteVar class. To build a program, clients declare an object of the Program class and invoke its constructor method.

Ideally, the client side for an instance of BATCHING would be exactly like the code of a client making direct calls to the server; i.e., like a client not using BATCHING at all. In practice, ConcreteControlStructure constructors (which are functions) are used. Thus, code in the client for a Program looks like the code that the user would write without using the pattern. Command objects are not declared; they are built with functional constructors.

To support the usage of expressions within the Program, subclasses inheriting from an Expr class can be provided (see Figure 6). Expr is a functional service representing an expression, and can be used as a function within expressions.

Program variables are stored in a table. They contain initial values as well as intermediate values and results of the program execution at the server side. To build that table, the programmer of the client must declare an object of the VarTable class. When variable objects are instantiated, they are constructed and stored in that table with an initial value, if any, and their mode, that is, in, out, inout, or local. When a variable table is sent to the server, only values of in and inout variables have to be copied to the server. After the execution of

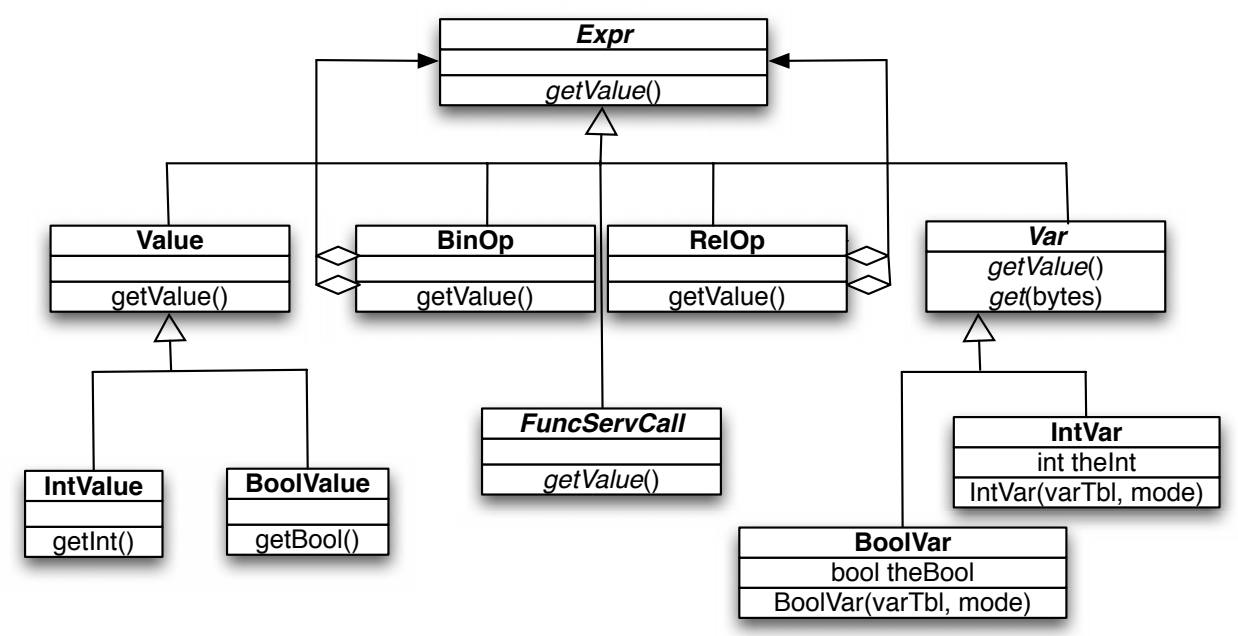

Fig. 6. Expression Hierarchy 
the program, out and inout variables are sent back to the client. Variables on the table can be consulted and modified on both sides.

The adequacy of the table implementation depends on the context of the pattern instance. For example, it can be interesting in an operating system to implement the table as a chunk of raw memory, while a remote server could represent it as a heterogeneous list of concrete variables.

This kind of implementation of BATCHING programs has the advantage that most of type-checking is done at compilation time. Note that server calls are type-checked, because the parameters of constructors of server call commands are typed. In a different context, however, a system designer may opt for a different implementation, for example, based on dynamic typing.

Revisiting our example, the code for the copy program is shown in Figure 7 In the figure, constructors are functions that build objects within the program. In this example, SeqCtrlStructure and WhileCtrlStructure are ConcreteControlStructures of the language. Open, Close, Read, and Write are classes derived from Program. Clients invoke their constructors to let the Program issue calls into the server. Program variables are stored in the vars variable table. In this case, $\mathbf{f} 1, \mathrm{f} 2$ and car are local variables, so their mode is local.

Finally, note that the concrete instruction set used through this section is just an example. Any other one, like a bytecode-based program could be used, too. Instruction sets suggested in the pattern are very simple compared to the ones used in other systems. For instance, $\mu$ Choices LTS ${ }^{+}$96 and Aglets PPE97 use a Java interpreter. A Modula-3 compiler is used in SPIN [BSP ${ }^{+}$95], and NetPebbles $\left[\mathrm{MPZ}^{+98}\right]$ uses a script interpreter.

When implementing the pattern, the design of the instruction set and its interpreter is one of the most important things the designer should keep in mind. The quality of the implementation depends heavily on the instruction set and interpreter being well-balanced and secure.

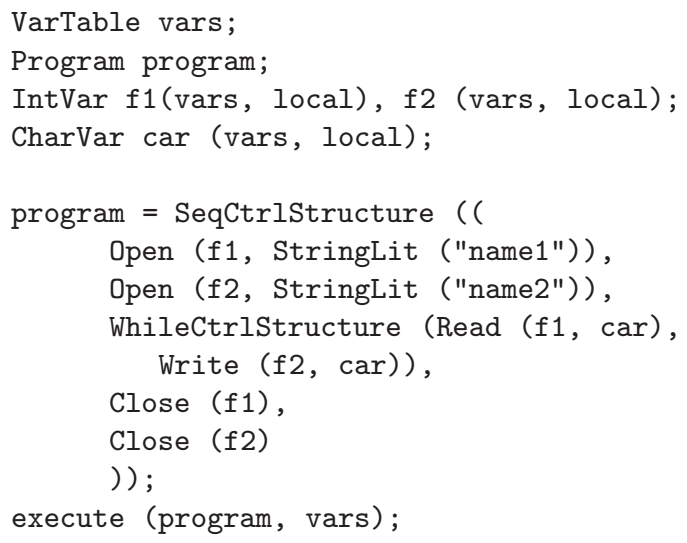

Fig. 7. Program for Copy 


\subsection{Exception Handling}

One of the problems of submitting the client code to the server is what happens when a call fails. The server programmer knows when a server call has failed, so he or she can decide to terminate the program in that case. This can be done by calling the terminate method of the Program class from a run method. However, the client could wish to continue the program despite any failures. To support this, we have included two commands in our pattern instances: AbortOnError and DoNotAbortOnError. They let the user switch between the two modes. When AbortOnError has been called, a call to terminate causes program termination; otherwise it has no effect. In this way, the client can control the effects of a failed call.

The implementation of terminate depends on both the kind of instruction set being implemented and on the implementation language. A bytecode-based program can be stopped very easily as there is a main control loop (in the run method), just by setting a terminated flag to true. Stopping a structured program (e.g., the one used in our file server example) is a little more complicated. This is due to recursive interpretation: calls to run in a Program propagate calls to the run method of its components. To stop that program, it is necessary to finish all the nested run calls. Depending on the implementation language, it can be done in one way or another. In a language with exceptions, such as $\mathrm{C}++$, Java or Python, it suffices to raise and propagate an exception in the terminate code, catching it in the Program run code. In languages without exceptions, such as $\mathrm{C}$, setjmp can be used in the top-level run method before calling any other run, and longjmp can be used, for the same purpose, in the terminate body.

\section{Consequences}

The pattern brings the following benefits:

1. It provides a virtual machine view of the server. When using BATCHING, clients no longer perceive servers as a separate set of entry points. Servers are now perceived as virtual machines [Nai05. Their instruction set is made of the set of server entry points, together with some general-purpose control language.

Therefore, it is feasible for users to reuse programs for different BATCHING calls. Programs that interact with the server can be built, and reused later.

2. It reduces protection-domain crossings, as the copy program did above. If this is the main motivation to use the pattern, domain crossing (client/server invocation) time must be carefully measured. Whenever complex control structures are mixed with calls to the server, or when client computations need to be done between successive calls, the pattern might not pay.

In any case, the time used to build the program must be lower than the time saved in domain crossing. The latter is approximately the difference between the time to perform a cross-domain call and the time to interpret and dispatch a server call. 
3. It reduces the number of messages exchanged by clients and servers; provided that the Program issues repeated calls to the server and the control structure is simple enough.

Again, the improvement due to the reduced number of messages can be lower than the overhead due to program construction and interpretation. Therefore, careful measurement must be done prior to pattern adoption.

4. It decouples client/server interaction from the call mechanism. BATCHING provides a level of indirection between the client and the server. The client can perform a call by adding commands to a Program; while the Program can be transmitted to the server by a means unknown to the client.

5. It decouples client calls from server method invocations. As said before, a client can perform calls by adding commands to a Program. The resulting Program can be sent to the server at a different time. Therefore, there is no need for the client and the server to synchronize for the call to be made.

6. It enables dynamic extension of servers. Servers can be extended by accepting Programs from clients. Those programs could be kept within the server and used as additional entry points into the server. Should it be the main motivation to use the pattern, the concrete command set should be powerful enough.

7. It makes the communication more secure because one can encrypt the complete sequence of commands in a single session, with a single signature. Many attack techniques rely on having a large quantity of messages to work with. Having a single message exchanged between the client and the server prevents variants of man-in-the-middle, replay, or other attacks GSS03 that would operate on individual commands if multiple messages were exchanged within a client/server communication session.

The pattern brings the following drawbacks:

1. Client requests might take arbitrary time to complete. A batched program might lead to a nonterminating program. If server correctness depends on bounded client requests, it may fail. As an example, a server can use a single thread of control to service all client requests. Should a Program not terminate, the entire server would be effectively switched off by a single client.

In such case, either avoid using BATCHING, or implement BatchServer with support for multithreading. That is, arrange for each Program to use its own thread. In this case, make sure the instruction set is thread-safe, otherwise programmers will need to rely on locking to protect critical, shared resources.

2. Server security can be compromised. The more complex the command set, the more likely the server integrity can be compromised due to bugs in the command interpreter. If high security is an issue, either avoid BATCHING or reduce the complexity of the command set to the bare minimum.

On the other hand, note that the pattern does not add functionality to the server. It simply enables the use of existing functionality in a "batch". Any server must always check its inputs (from clients) and these checks must still be performed when the individual calls come from the pattern interpreter. 
3. It might slow down the application. When cheap domain crossing is available and efficiency is the primary objective, using BATCHING might slowdown the application if the time saved on domain crossings is not enough to compensate for the overhead introduced by BATCHING.

4. Clients might become more complex because they must build the program to be sent, instead of simply issuing the calls to the server when they are needed.

\section{Related Patterns}

Both Program and ControlStructure rely on instances of the INTERPRETER pattern GHJV95. Indeed, the interpreter of a Program is behind its run method.

Program, ControlStructure, and Commands make up an instance of the CoMPOSITE pattern GHJV95. Composite programs, such as sequence and Conditional, are aggregates of Assignments, ServerCalls, and other primitive commands.

If an instruction set for a BATCHING language is to be compiled, Program might include a method to compile itself into a low-level instruction set. Moreover, Programs should be serialized (and later deserialized) when transmitted to the server. Once in the server, they can be verified for correctness. All these tasks can be implemented following the VISITOR pattern GHJV95.

A server call issued within a Program might fail or trigger an exception. If that is the case, the entire Program can be aborted and program state transmitted back to the client-so that the client could fix the cause of the error and resume Program execution. The Memento pattern GHJV95 can encapsulate the program state while in a "frozen" state. As said before, such program state can be used to resume the execution of a failed program (e.g., after handling an exception). Mementos can also be helpful for (de)serializing the program during transmission to the server.

As a program can lead to an endless client request, single threaded or arequest-at-a-time servers can get into trouble. To accommodate this kind of server so that BATCHING could be used, the ActiveOBJECT [LS95] and the RENDEZVous [JPPMA99] patterns can be used.

Compositemessages can be used to transfer the Program from the client to the server. The CompositeMessages pattern [SC95] applies when different components must exchange messages to perform a given task. It groups several messages in a structured fashion, doing with messages what BATCHING does with server entry-points. In that way, extra latency due to message delivery can be avoided and components are decoupled from the transmission medium. The main difference is that BATCHING is targeted at the invocation of concrete server-provided services, not at packaging data structures to be exchanged.

ComposedCommand Tid98 is similar to BATCHIng in that it bundles several operations into a single one. However, BATCHING is more generic in spirit.

Adaptive OBJect-Models YJ02 is an architectural style in which the users' object model is interpreted at runtime and can be changed with immediate 
effects on the system interpreting it. It is normally seen in advanced commercial systems in which business rules are stored in places such as databases or XML files. Although its implementation might resemble the BATCHING pattern, its major goal is to provide more flexibility and enable runtime reconfiguration of business rules and not to improve the performance.

\section{Known Uses}

Our experience with BATCHING started when we noticed that a single piece of design had been used to build systems we already knew well. Then we tried to abstract the core of those systems, extracting the pattern. Once we identified the pattern, we tried to find some new systems where it could be applied to obtain some benefit. We did so $\mathrm{BJP}^{+} 00$ and obtained substantial performance improvements.

For us, this pattern has been a process where we first learned some "theory" from existing systems and then applied what we learned back to "practice." In this section, we show how existing systems match the pattern described in the previous sections - certainly, this will lead to a better understanding of the pattern, as happened in our case. We also include a brief overview of the two systems where we applied the pattern ourselves with a priori knowledge of the pattern.

Note that the BATCHING design lets a single implementation of the pattern handle the various applications described below. As the activity carried out at the server is specified every time a Program runs, the same BATCHING implementation could perfectly handle most of the applications shown below. Nevertheless, existing systems, built without a priori knowledge of the pattern, hardly share the common code needed to implement all these applications (e.g., gather/scatter is always implemented separately from message batching facilities, when both are provided.)

Operating System extensions by downloading code into the kernel (as performed in SPIN $\mathrm{BSP}^{+}$95], $\mu$ Choices $\left[\mathrm{LTS}^{+}\right.$96], and Linux Hen06]) can be considered to be an instance of this pattern. These systems use code downloading as the means to extend system functionality. The mechanism employed is based on defining new programs, which are expressed in terms of existing services.

In this case the Program is the extension performed, the set of ConcreteControlStructures depends on the extension language, and the run method is implemented either by delegation to the extension interpreter or by the native processor (when binary code is downloaded into the system.)

Agents. An agent is a piece of autonomous code that can be sent to a different domain. Agents may move from one domain to another, carrying its runtime state BR05. The aim is to avoid multiple domain crossings (or network messages), improving performance, and support disconnection from the agent home environment. 
Programs built using BATCHING are meant to stay at the server until termination, and they possess no gd2 ${ }^{2}$ statement. However, BATCHING already includes most of the machinery needed to implement an agent system; a go statement could be provided by the command language itself. Nevertheless, even within the mobile agent paradigm, a very common situation is to have a single-hop agent that leaves a client, visits a single server and return to the client, as is the case with BATCHInG.

Gather/Scatter I/O. In gather/scatter I/O a list of input or output descriptors is sent to an $\mathrm{I} / \mathrm{O}$ device in a single operation. Each descriptor specifies a piece of data going to (or coming from) the device. Written data is gathered from separate output buffers. Read data is scattered across separate input buffers. Its major goal is to save data copies.

In this case, the program is just the descriptor list, where each descriptor can be supported by a Command. The program run method iterates through the descriptor (i.e., command) list and performs the requested I/O operations. The services (i.e., commands) are simply Read and Write.

Note that, by using this pattern, gather/scatter I/O could be generalized so that the I/O device involved would not necessarily be the same for all descriptors sent by the user. Moreover, multiple Read and Write operations could be bundled into a single one.

Message batching. Grouping a sequence of messages into a single low-level protocol data unit is yet another instance of the pattern. In this case, the run method (i.e., the interpreter) is the packet disassembler. A program is a bunch of packets bundled together. Each packet, or each packet header, is a command that is interpreted by the packet disassembler. This is the BATCHInG application that more closely resembles ComposedCommand Tid98.

Deferred calls. BATCHING can be used to support disconnected operation MRM06. Clients build programs while they perform operations on nonreachable servers whenever they are in a disconnected state. Upon reconnection, each program is finally submitted to the target domain for interpretation. Note that several clients might add code to a single program to be sent to the server later on.

Each operation is a Command, the list of operations sent to a server is a Program. The interpreter could be either:

1. the piece of code sending each command when the client is reconnected to the server, or

2. an actual Program interpreter in the server domain, accepting just a list of commands (a program) - to save network traffic.

Futures or Promises LS88] can be used by client code to synchronize with server responses.

Improving latency in Operating Systems. Many user programs happen to exhibit very simple system call patterns. This is an opportunity for using

${ }^{2}$ The go instruction is typical on Agent systems and is meant to trigger the migration of an agent to a different location. 
BATCHING to save domain crossings and, therefore, execution time. As a matter of fact, we have done so by instantiating BATCHING for two systems: Linux and Off ++ $\mathrm{BHK}^{+99}$. In both systems, we obtained around $25 \%$ speedups for a copy program written with BATCHInG $\mathrm{BJP}^{+} 00$.

We implemented two new domain-specific languages (i.e., ControlStructures and Command sets) that let users bundle separate calls into a single one, like in the copy example of Section 1. The first language we implemented was based on bytecodes. We included just those commands needed to code loops, conditional branches, and basic arithmetic. This language was used both on Linux and $O f f++$. The second language we implemented was a high-level one, designed specifically for $O f f++$. It includes just the commands needed to Repeat a given operation $\mathrm{n}$ times and to perform a Sequence of operations $\left[\mathrm{BJP}^{+} 00\right]$.

Heterogeneous resource allocation. Most operating systems are structured as a set of resource unit providers. Separate servers provide resource unit allocation for different types of resources. In these systems, users issue multiple requests at a time.

BATCHING can be used to request allocation of multiple heterogeneous resources in a single system call. Off ++ is an operating system modeled as a set of hardware resource unit providers and it uses BATCHING in this way to improve the performance of its applications $\mathrm{BJP}^{+} 00$.

Transaction processing. A transaction is a set of operations executed atomically in isolation Gra78. A given transaction can either terminate normally, by committing, or abnormally, by aborting. Should a transaction abort, its effects must be undone; otherwise (i.e., when it commits), its results should be made permanent.

Commits typically involve multiple disk writes for different data items. Writes must follow a carefully chosen order to preserve the consistency of results, even when failures occur. One of the strategies uses a redo algorithm BHG87. Such algorithm does not modify the persistent data until the transaction is completed, it works on a volatile copy of the data until the commit is performed. At commit time, a sequence of redo records is written into a disk log, followed by a commit record. Redo records contain new values for objects changed by the transaction. Finally, persistent state for objects involved is updated. If the system fails before the write of the commit record, the transaction is aborted and their redo records are ignored. If the system fails after writing the commit record, redo records are replayed.

Another instance of BATCHING is group commit $\left[\mathrm{DKO}^{+84}\right]$, used to avoid the latency of forced disk writes of log records in each commit. In group commit, instead of forcing the log with each commit, a set of consecutive commits are batched. Then, a single forced disk write is performed to write all the $\log$ records associated with all the commits, amortizing the latency of forced disk writes across several commits. This results in a substantial improvement in throughput for commits. 
Batching can be used both to implement commit and for crash recovery. Performance of transactional distributed object systems (e.g., Arjuna [SDP91]) could be improved due to the reduced number of domain crossings.

\section{Variant}

A widely-used variant of this pattern is the CLIENT-SIDE BATCHING pattern. In this case, instead of the client sending batched code to be executed in the server, it is the server that sends code to be executed in the client.

The Client-SIDE BATChing pattern is frequently used on the Web where the inter-domain communication latency is normally very large. Known uses include Java applets Boe02, Javascript functions embedded in Web pages Fla02, and Macromedia Flash applications Abe02.

In both BAtching and Client-Side BAtching, the goal is to improve the response time of the system as perceived by the client and, in both cases, this is achieved by avoiding multiple cross-domain calls. The difference is where the batched program is executed, in the client or in the server.

\section{Conclusions}

BATChing unifies several, apparently unrelated, techniques. Most notably, the pattern integrates techniques to (1) reduce domain crossings and (2) avoid unnecessary data copying. Encapsulation of the command language has been a key feature in the integration of existing techniques, decoupling the command set from the submission method.

We showed eight different applications where the BATCHING pattern was previously used and cases where the pattern was applied with a-priori knowledge of it. A client-side variant of the pattern is also implemented by different technologies and is widely-used on current Web systems.

\section{Acknowledgments}

We are sincerely grateful for the help provided by our shepherd, Frank Buschmann, and for the valuable feedback provided by John Vlissides, who suggested the new name for this pattern (it was previously called CompositeCALL). Finally, we are also grateful to the anonymous TPLoP reviewers and to the members of the "Allerton Patterns Project" group of PLoP'99 for their comments and suggestions.

\section{References}

[Abe02] Aberdeen Group: Flash Remoting MX: A Responsive Client-Server Architecture for the Web. Technical report, Macromedia White paper (December 2002)

[BHG87] Bernstein, P.A., Hadzilacos, V., Goodman, N.: Concurrency Control and Recovery in Database Systems. Addison-Wesley, Reading (1987) 
[BHK ${ }^{+99] \quad B a l l e s t e r o s, ~ F . J ., ~ H e s s, ~ C ., ~ K o n, ~ F ., ~ A r e ́ v a l o, ~ S ., ~ C a m p b e l l, ~ R . H .: ~ O b j e c t ~}$ Orientation in Off ++ - A Distributed Adaptable $\mu$ Kernel. In: Proceedings of the ECOOP 1999 Workshop on Object Orientation and Operating Systems, pp. 49-53 (1999)

$\left[\right.$ BJP $\left.^{+} 00\right]$ Ballesteros, F.J., Jimenez, R., Patino, M., Kon, F., Arévalo, S., Campbell, R.H.: Using Interpreted CompositeCalls to Improve Operating System Services. Software: Practice and Experience 30(6), 589-615 (2000)

[Boe02] Boese, E.S.: Java Applets: Interactive Programming, 2nd edn. Lulu.com (2002)

[BR05] Braun, P., Rossak, W.: Mobile Agents: Basic Concepts, Mobility Models, and the Tracy Toolkit. Elsevier, Amsterdam (2005)

$\left[\mathrm{BSP}^{+} 95\right]$ Bershad, B.N., Savage, S., Pardyak, P., Sirer, E.G., Fiuczynski, M., Becker, D., Eggers, S., Chambers, C.: Extensibility, safety and performance in the SPIN operating system. In: Proceedings of the Fifteenth ACM Symposium on Operating Systems Principles, December 1995, ACM, New York (1995)

$\left[\mathrm{DKO}^{+} 84\right]$ DeWitt, D.J., Katz, R.H., Olken, F., Shapiro, L.D., Stonebraker, M.R., Wood, D.: Implementation techniques for main memory database systems. In: Proceedings of the ACM International Conference on Management of Data (SIGMOD), pp. 1-8 (1984)

[Fla02] Flanagan, D.: JavaScript: the definitive guide. O'Reilly, Sebastopol (2002)

[GHJV95] Gamma, E., Helm, R., Johnson, R., Vlissides, J.: Design Patterns. Elements of Object-Oriented Software. Addison-Wesley, Reading (1995)

[Gra78] Gray, J.: Operating Systems: An Advanced Course. Springer, Heidelberg (1978)

[GSS03] Garfinkel, S., Spafford, G., Schwartz, A.: Practical UNIX and Internet Security. O’Reilly, Sebastopol (2003)

[Hen06] Henderson, B.: Linux Loadable Kernel Module HOWTO. Technical report, Linux Documentation Project (September 2006)

[JPPMA99] Jiménez-Peris, R., Patiño-Martínez, M., Arévalo, S.: Multithreaded Rendezvous: A Design Pattern for Distributed Rendezvous. In: Proc. of ACM Symposium on Applied Computing, February 1999, ACM Press, New York (1999)

[LS88] Liskov, B., Shrira, L.: Promises: Linguistic Support for Efficient Asynchronous Procedure Calls in Distributed Systems. In: Proc. of ACM Conf. on Programming Language Design and Implementation, pp. 260267 (1988)

[LS95] Lavender, R.G., Schmidt, D.C.: Active object - an object behavioral pattern for concurrent programming. In: Proceedings of the Second Pattern Languages of Programs conference (PLoP), Monticello, Illinois (September 1995)

[LTS ${ }^{+96}$ Li, Y., Tan, S.M., Sefika, M., Campbell, R.H., Liao, W.S.: Dynamic Customization in the $\mu$ Choices Operating System. In: Proceedings of Reflection 1996, San Francisco (April 1996)

[MPZ ${ }^{+}$98] Mohindra, A., Purakayastha, A., Zukowski, D., Devarakonda, M.: Programming Network Components Using NetPebbles: An Early Report. In: Proceedings of the 4th USENIX Conference on Object-Oriented Technologies and Systems, Santa Fe, New Mexico (April 1998) 
[MRM06] Mikic-Rakic, M., Medvidovic, N.: A Classification of Disconnected Operation Techniques. In: Proceeding of 32nd EUROMICRO Conference on Software Engineering and Advanced Applications (EUROMICRO 2006), pp. 144-151. IEEE Computer Society, Los Alamitos (2006)

[Nai05] Nair, R.: Virtual Machines: Versatile Platforms for Systems and Processes. Morgan Kaufmann, San Francisco (2005)

[PPE97] Clements, P.E., Papaioannou, T., Edwards, J.: Aglets: Enabling the Virtual Enterprise. In: Proc. of the Managing Enterprises - Stakeholders, Engineering, Logistics and Achievement Intl. Conference (ME-SELA 1997), Loughborough University, UK (1997)

[SC95] Sane, A., Campbell, R.: Composite Messages: A Structural Pattern for Communication between Components. In: OOPSLA 1995 workshop on design patterns for concurrent, parallel, and distributed object-oriented systems (1995)

[SDP91] Shrivastava, S.K., Dixon, G.N., Parrington, G.D.: An Overview of Arjuna: A Programming System for Reliable Distributed Computing. IEEE Software 8(1), 63-73 (1991)

[Tid98] Tidwell, J.: Interaction Design Patterns. In: Proceedings of the Conference on Pattern Languages of Programs (PLoP 1998), Monticello, Illinois (1998)

[YJ02] Yoder, J.W., Johnson, R.: The Adaptive Object Model Architectural Style. In: Proceeding of The Working IEEE/IFIP Conference on Software Architecture 2002 (WICSA3 2002). Kluwer Academic Publishers, Dordrecht (2002) 